\title{
Novel Drug Delivery Systems and its Future Prospects
}

\author{
Avani K. Shewale ${ }^{1 *}$, Akshay R. Yadav², Ashwini S. Jadhav ${ }^{3}$ \\ ${ }^{1}$ Assistant Professor Department of Pharmacology, Rajarambapu College of Pharmacy, \\ Kasegaon, Maharashtra, India-415404 \\ ${ }^{2}$ Assistant Professor Department of Pharmaceutical Chemistry, Rajarambapu College of \\ Pharmacy, Kasegaon, Maharashtra, India-415404 \\ ${ }^{3}$ Assistant Professor, Department of Pharmaceutics, Rajarambapu College of Pharmacy, \\ Kasegaon, Dist-Sangli, Maharashtra, India-415404
}

\begin{abstract}
Most common methods of delivery include the preferred topical (skin), transmucosal (nasal, buccal, sublingual, vaginal, ocular and rectal) and inhalation routes. The conventional dosage forms provide drug release immediately and it causes fluctuation of drug level in blood depending upon dosage form. Therefore to maintain the drug concentration within therapeutically effective range needs novel drug delivery system. In the past few decades, considerable attention has been focused on the development of novel drug delivery system (NDDS). The NDDS should ideally fulfill two prerequisites. Firstly, it should deliver the drug at a rate directed by the needs of the body, over the period of treatment. Secondly, it should channel the active entity to the site of action. In conventional drug delivery systems, there is little or no control over release of the drug and effective concentration at the target site can be achieved by irregular administration of grossly excessive doses. At present, no available drug delivery system behaves ideally, but sincere attempts have been made to achieve them through various novel approaches in drug delivery.
\end{abstract}

Keywords: Novel drug delivery system, Drug delivery carriers, Phytosomes, Aquasomes, Sphinosomes, Transferosomes.

\section{INTRODUCTION}

Novel Drug Delivery System (NDDS) refers to the approaches, formulations, technologies, and systems for transporting a pharmaceutical compound in the body as needed to safely achieve its desired therapeutic effects ${ }^{1}$. It may involve scientific site-targeting within the body, or it might involve facilitating systemic pharmacokinetics; in any case, it is typically 
concerned with both quantity and duration of drug presence. NDDS aims at providing some control, whether this is of temporal or spatial nature or both, of drug release in the body. Novel drug delivery attempts to either sustain drug action at a predetermined rate, or by maintaining a relatively constant, effective drug level in the body without concomitant minimization of undesirable side effects. It can also localize drug action by spatial placement of controlled release systems adjacent to, or in the diseased tissue or organ; or target drug action by using carriers or chemical derivatization to deliver drug to particular cell type $\mathrm{e}^{2}$. A number of NDDS have emerged encompassing various routes of administration, to achieve controlled and targeted drug delivery. Encapsulation of drug in vesicular structure is one such system, which can be predicted to prolong the existence of the drug in systemic circulation, and reduce the toxicity, if selective uptake can be achieved ${ }^{3}$.

\section{Advantages of Novel Drug Delivery Systems ${ }^{4-5}$}

1. Reduce the number and frequency of doses required to maintain the desired therapeutic response.

2. Reduction in the total amount of drug administered over the period of drug treatment.

3. Reduced blood level oscillation characteristic of multiple dosing of conventional dosage forms.

4. Reduction in the incidence and severity of both local and systemic side effects related to high peak plasma drug concentration.

5. Protection from first pass metabolism and gastro intestinal tract degradation.

6. Maximizing availability with minimum dose.

7. Safety margin of high potency drugs can be increased.

8. Targeting the drug molecule towards the tissue or organ reduces the toxicity to the normal tissues.

9. Improved patient compliance. 
$>$ Increased efficacy of the drug.

$>$ Site specific delivery.

$>$ Decreased toxicity / side effects.

$>$ Increased convenience.

$>$ Shorter hospitalization.

$>$ Viable treatments for previously incurable diseases.

$>$ Potential for prophylactic application.

$>$ Lower health care costs- both short and long term.

$>$ Better patient compliance.

Novel drug delivery systems can include those based on physical mechanisms and those based on biochemical mechanisms. Physical mechanisms also referred as controlled drug delivery systems include osmosis, diffusion, erosion, dissolution and electro transport. Biochemical mechanisms include monoclonal antibodies, gene therapy, and vector systems, polymer drug addicts and liposomes. Therapeutic benefits of some new drug delivery systems include optimization of duration of action of drug, decreasing dosage frequency, controlling the site of release and maintaining constant drug levels. Among drug carriers one can name soluble polymers, microparticles made of insoluble or biodegradable natural and synthetic polymers, microcapsules, cells, cell ghosts, lipoproteins, liposomes, and micelles. The carriers can be made slowly degradable, stimuli-reactive (e.g. pH- or temperature sensitive), and even targeted (e.g., by conjugating them with specific antibodies against certain characteristic components of the area of interest). Targeting is the ability to direct the drugloaded system to the site of interest. Two major mechanisms can be distinguished for addressing the desired sites for drug release:

(i) Passive and

(ii) (ii) Active targeting ${ }^{6-8}$. 


\section{Drug Delivery Carriers}

Carriers are used to achieve targeted drug delivery. Carrier is one of the special molecule or system essentially required for effective transportation of loaded drug up to the preselected sites. They are engineered vectors, which retain drug inside or onto them either via encapsulation and or via spacer moiety and transport or deliver it into vicinity of target cell ${ }^{9-}$ 10 .

Some carrier based drug delivery systems are,

Microspheres and micro capsules

Nanoparticles

Monoclonal antibodies

Prodrugs

Resealed erythrocytes

Artificial cells

Neutrophils

Vesicular carriers

\section{* Vesicular systems}

In recent years, vesicles have become the vehicle of choice in drug delivery. Lipid vesicles were found to be value of immunology, membrane biology, diagnostic techniques, and most recently, genetic engineering. Vesicles can play a major role in modelling biological membranes, and in the transport and targeting of active agents. Biological membranes form the ubiquitous delimiting structures that surround and compartmentalize all cells and organelles. The bilayer arrangement of lipids is perhaps the only organizational feature that 
is common to all biological membranes. Numerous theoretical models of membrane structure have appeared since the publication of the cell theory by Schleiden and Sehwann in 1839. Experimental models provide insight into the national dynamics and static structures of some isolated compartments of biological membranes. Lipid vesicles are just one type of many experimental models of biomembranes. Although developed for basic research, many technological innovations have arisen from the applications of these models. Lipid vesicles have evolved successfully, as vehicles for controlled drug delivery ${ }^{11}$.

Some important vesicular drug delivery systems are,

Phytosomes

Aquasomes

Liposomes

Sphinosomes

Transferosomes

Niosomes

Ethosomes

\section{$>$ Phytosomes}

Phytosomes are one of the novel drug delivery system containing hydrophilic bioactive phytoconstituents of herbs surround and bound by phospholipids. This phyto-phospholipid complex resembles a little cell which exhibit better pharmacokinetic and pharmacodynamic profile than the conventional herbal extract resulting in better bioavailability. There is an enhancement of the bioavaibility of botanical extract due to their complexation with phospholipid and improved absorption in the intestinal tract. The formulation of phytosomes is safe and the components have all been approved for pharmaceuticals and cosmetic use. Phytosomes shows better stability profile because chemical bond are formed between 
phosphotidylcholine molecules and phytoconstituents (shown in Fig.1) ${ }^{12-13}$.

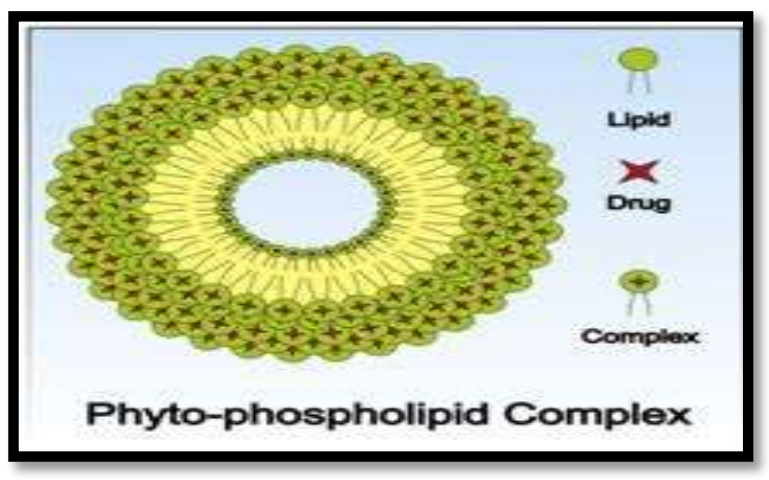

Fig 1. Structure of phytosomes

\section{$>$ Aquasomes}

Aquasomes are one of the most recently developed delivery system for bioactive molecules like peptide, protein, hormones, antigens and genes to specific sites. Aquasomes are spherical in shape with 60-300 nm particles size. These are nanoparticulate carrier systems but instead of being simple nanoparticles these are three layered self assembled structures, comprised of a solid phase nanocrystalline core coated with oligomeric film to which biochemically active molecules are adsorbed with or without modification. These structures are self assembled by non covalent and ionic bonds. The solid core provides the structural stability, while the carbohydrate coating protects against dehydration and stabilizes the biochemically active molecules. The delivery system has been successfully utilized for the delivery of insulin, hemoglobin, and enzymes like serration peptidase etc ${ }^{14-15}$.

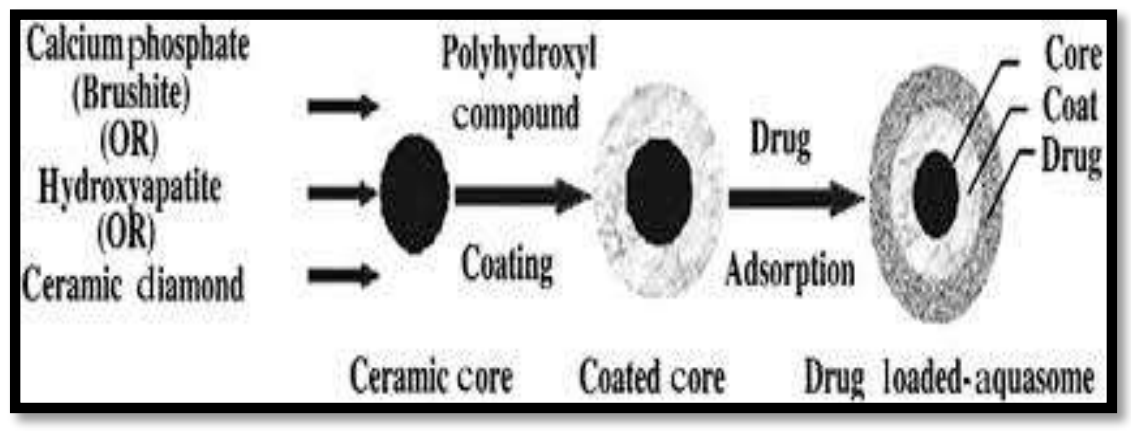

Fig 2. Structure of aquasomes 


\section{$>$ Liposomes}

Liposomes (meaning lipid body) are spherical microscopic vesicles compose of one or more concentric lipid bilayers, separated by water or aqueous buffer compartments with a diameter ranging from $25 \mathrm{~nm}$ to $10000 \mathrm{~nm}$. Self assembly of phospholipid molecules in an aqueous environment results in the formation of liposomes. They are commonly composed of one or more amphiphilic phospholipid bilayer membranes (and thus also called as phospholipid vesicles) that can entrap both hydrophilic and hydrophobic drugs. Hydrophilic drugs are entrapped in the aqueous centre of liposome while the liposome wall, being phospholipid membrane can hold hydrophobic agents. There are a number of components present in liposomes, with phospholipids and cholesterol being main ingredients. The phospholipids used for making liposomes include phosphatidylcholines (PC), phosphatidylethanolamines (PE) and phosphatidylserines (PS). Besides, phospholipids, sphingolipids, glycolipids and sterols can also be used to prepare liposomes. These vesicles are exploited to achieve altered drug pharmacokinetics and targeted therapies ${ }^{16-17}$.

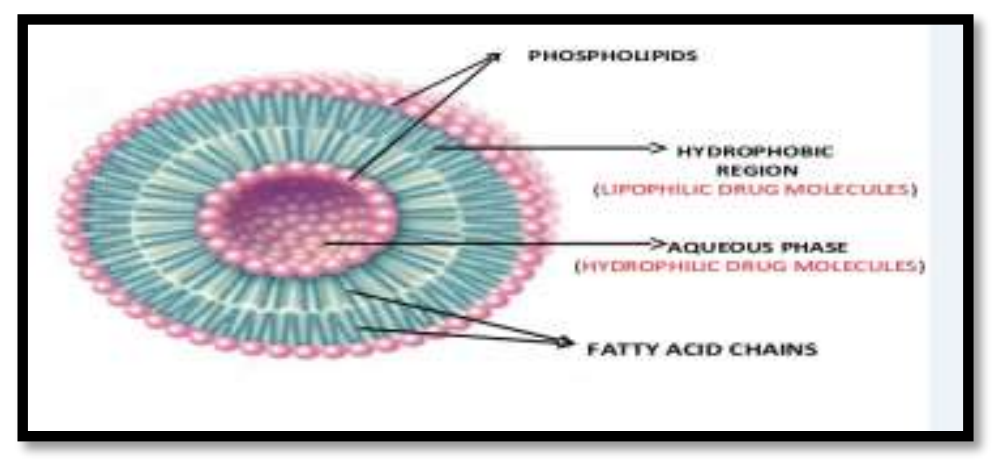

Fig 3. Structure of Liposomes

\section{$>$ Sphinosomes}

Sphingosome may be defined as "concentric, bilayered vesicle in which an aqueous volume is entirely enclosed by a membranous lipid bilayer mainly composed of natural or synthetic sphingolipid. Sphingosomes are administered in many ways these include parentral route of administration such as intravenous, intramuscular, subcutaneous, and intra-arterial. 
Generally it will be administered intravenous or some cases by inhalation. Often it will be administered into a large central vein, such as the superior vena cava and inferior vena cava to allow highly concentrated solution to be administered into large volume and flow vessels. Sphingosomes may be administered orally or transdermally. In simple way we can say sphingosome is liposome which is composed of sphingolipid ${ }^{18-19}$.

\section{Transferosomes}

Transfersomes is latest novel drug delivery system and are special types of liposomes, consist of phosphatidylcholine and an edge activator. They are ultra flexible membrane, which deliver the drug reproducibly either into or through the skin. The system delivers the drug with high efficiency depending on the choice of administration or application. This system has several order magnitude of elasticity and flexibility over liposomal drug delivery which makes it favourable for efficient skin penetration and hence for the novel drug delivery system. Transferosomes is a highly adaptable and optimized mixed lipid complex aggregate. The transferosomes crossvarious transport barriers efficiently and then act as a drug carrier for non invasive targeted drug delivery and sustained release of therapeutic agents. Transferosomes are super molecular entities that can pass through a permeability barrier and there by transport material from the site of application to the destination. The transferosomes enhance the permeation of most of low as well as high molecular weight drugs ${ }^{20-21}$.

\section{Niosomes}

Niosomes are a novel drug delivery system, in which the medication is encapsulated in a vesicle. The vesicle is composed of a bilayer of non-ionic surface active agents and hence the name niosomes. The niosomes are very small, and microscopic in size. Their size lies in the nanometric scale. Although structurally similar to liposome's, they offer several advantages over them. Niosomes have recently been shown to greatly increase transdermal drug delivery 
and also can be used in targeted drug delivery, and thus increased study in these structures can provide new methods for drug delivery. In recent years, niosomes have been extensively studied for their potential to serve as a carrier for the delivery of drugs, antigens, hormones and other bioactive agents. Besides this, noisome have been used to solve the problem of insolubility, instability and rapid degradation of $\operatorname{drugs}^{22}$.

\section{Ethosomes}

"Ethosomes are ethanolic liposomes". Ethosomes can be defined as noninvasive delivery carriers that enable drugs to reach deep into the skin layers and/or the systemic circulation. These are soft, malleable vesicles tailored for enhanced delivery of active agents. The vesicles have been well known for their importance in cellular communication and particle transportation for many years. Vesicles would also allow controlling the release rate of drug over an extended time, keeping the drug shielded from immune response or other removal systems and thus be able to release just the right amount of drug and keep that concentration constant for longer periods of time $\mathrm{e}^{23-25}$.

\section{* Future of Nanomedicine and Drug Delivery System}

Nanomedicine science is one of the most promising fields of study at the present time. In the last two decades, a great deal of research in this area has already resulted in 1500 patents being filled and several dozen clinical trials completed. While the potential prospect of nanomedicine and nanodrug delivery is generally known, the true effect of the nanomedicine system on the health system is still very small, including in cancer therapy and diagnoses. This is a new area of science, and only two decades of true study on this topic are still unknown in the field, with several primary fundamental attributes. One major area of research in the future is the fundamental markers of diseased tissues including key biological markers which allow for absolute targeting without changing the normal cell 
process. Finally, with our growing understanding of molecular diseases or a comparable identification for markers with a nanomaterial-subcellular scale, the application of nanomedicine will lead to new diagnosis/therapy. The understanding of disease's molecular signatures would therefore contribute to more advancement in applications of nanomedicine. Further research will be crucial for further application of nanomedicine beyond what has been mentioned in this analysis with the known nanoprobes and products from nanotheragnostics. The principle of controlled release of specific medication in bellicose areas has not yet been mastered, the technology used to test these events, the effect of the medicines in the tissue/cell level and theoretical mathematical prediction models. Numerous research projects in the areas of nanomedicine focus on biomaterials and formulation studies which tend to be the first stage in applications for biomedicine. Valuable proof will come from animal trials and multidisciplinary studies requiring considerable time and testing resources in future applications such as medical therapies and Diagnostic Studies. With a rising global movement towards more accurate medicines and diagnostics, nano-medicine and nano-drug delivery technologies look better for the future ${ }^{26-27}$.

\section{CONCLUSION}

The new method of delivery not only eliminates the repeated administration to overcome non-compliance, but also helps to improve therapeutic benefit through the reduction of toxicity, bioavailability and so on. Extensive research is under way into new drug delivery methods for herbal medicinal products. Implementation of these revolutionary techniques in natural medicines would increase bioavailability, decrease toxicity, sustained release action, GI safety not obtained through the traditional molecular delivery system, low solubility, herbal drug degradation in Gas

\section{REFERENCES}


1. Bhagwat R, Vaidhya I, Novel drug delivery system: an overview, Int J Pharm Sci Res. 2013; 4(3): 970-982 .

2. Chien Y, Novel drug delivery systems, Marcel Dekkar. Inc, 1992, Revised 2nd edition, 1133.

3. Vyas $S$, Khar R. Targeted and controlled drug delivery, novel carrier systems, 2002; 1:3946.

4. Patil S, Mhaiskar A, Mundhada D. A Review On Novel Drug Delivery System: A Recent Trend. Int J Cur Pharm \& Clin Res. 2016 ;6(2): 89-93.

5. Kumar K, Rai AK. Miraculous therapeutic effect of herbal drug using novel drug delivery system, Intl Res J Pharm. 2012;3(2): 27-30.

6. Duvaz I. C.J. Antibody-directed enzyme prodrug therapy (ADEPT): a review, Adv Drug Delivery Rev. 1997; 26: 151-72.

7. Manabe T, Okino H, Maeyama R, Mizumoto K, Nagai E, Tanaka M, Matsuda T. Novel strategic therapeutic approaches for prevention of local recurrence of pancreatic cancer after resection: transtissue, sustained local drug-delivery systems. J Controlled Release. 2004; 100: 317-30.

8. Ziaie B, Baldi A, Lei M, Gu Y, Siegel RA. Hard and Soft Micro machining for Biomems. Review of Techniques and Examples of Applications in Microfluidics and Drug Delivery, Adv Drug Delivery Rev. 2004; 56: 145-72.

9. Agnihotri J, Saraf S, Khale A. Targeting: New potential carriers for targeted drug delivery system, Int J Pharm Sci Rev Res. 2011; 8(2): 117-122.

10. P. Dwarakanadha Reddy, D. Swarnalatha, Recent Advances in Novel Drug Delivery Systems, Int J Phaml Technol Res. 2010; 2(3):2025-2027.

11. Kumar R, Kumar S, Shankar S, Jha A. Vesicular System-Carrier for Drug Delivery, Der Pharmacia Sinica. 2011; 2(4): 192-202. 
12. Sharma S, Sikarwar M,Phytosome: A review, Plant indica, 2005; 1(2): 1-3.

13. Gupta A, Ashawat MS, Saraf S, Phytosome: A novel approach towards functional cosmetics, J. Plant Sci. 2007; 2(6): 644-649.

14. Goyal A.K., Khatri K., Mishra N., Mehta A., Vaidya B., Tiwari S, Development of selfassembled nanoceramic carrier construct(s) for vaccine delivery, J. Biomater Application, 2009; 24: 65-84.

15. Khopade A.J., Khopade S., Jain N.K, Development of haemoglobin aquasomes from spherical hydroxyapatite cores precipitated in the presence of poly(amidoamine) dendrimer. Drug Dev Ind Pharm. 2002; 241:145-54.

16. Sharma A, Sharma. U.S, Liposomes in drug delivery: progress and limitations, Int J Pharm. 1997; 154: 123-140.

17. Nagarsenker M, Londhe V. Nadkarni G. Preparation and evaluation of liposomal formulations of tropicamide for ocular delivery. Int J Pharm. 1999; 190(1): 63-71.

18. Ashok K, Nama R. Sphingosomes: A novel vesicular drug delivery system. Int J Pharm Res \& bio-Sci. 2013; 2(2): 2277-8713.

19. Tejasavi M, Sonica P, Tania S, Sunil K, Sohit M, Neeraj B. Vesicular Drug Delivery System: A Review, World J Pharm Pharm Sci. 2018;7(10):667-679.

20. Yadav A, Mohite S. Applications of Nanotechnology in Cosmeceuticals. Research J. Topical and Cosmetic Sci. 2020; 11(2): 83-88.

21. Yadav A, Mohite S. Potential Role of Peptides for Development of Cosmeceutical skin Product. Research J. Topical and Cosmetic Sci. 2020; 11(2): 77-82.

22. Suryawanshi V, Yadav A, Birajdar R, Jagtap N, Vambhurkar G, Patil P. Optimization of ayurvedic herbal medicine by nanoformulation. Asian J. Res. Pharm. Sci. 2019; 9(1): 5556. 
23. Kumavat S, Chaudhari $Y$, Borole P, Duvvuri P, Bubera $N$, Shenghani $K$, Shah $P$. Transferosomes: A Promising approach for transdermal drug delivery system, Asian Journal of Pharmaceutical Sciences and Research, 2013; 3(5): 2249 - 4898.

24. Yadav A, Mohite S. Aquasomes as a Self Assembling Nanobiopharmaceutical Carrier System for Bio-Active Molecules. Research J. Topical and Cosmetic Sci. 2020; 11(2): 6670.

25. Yadav A, Mohite S. Different Techniques and Characterization of Polymorphism with their Evaluation: A Review. Asian J. Pharm. Tech. 2020; 10(3): 213-216

26. Kumavat $S$, Chaudhari $Y$, Borole $P$, Duvvuri $P$, Bubera $N$, Shenghani $K$, Shah $P$. Transferosomes: A Promising approach for transdermal drug delivery system, Asian J Pharm Sci Res. 2013 ;3(5): 2249 - 4898.

27. Buchiraju R, Nama S, Sakala B, Chandu B, Kommu A. Vesicular Drug Delivery System An Overview, Res. J Pharm Biol Chem Sci. 2013; 4(3): 462-466. 